\title{
Deciding Nonconstructibility of 3-Balls with Strict Spanning Edges
}

\author{
Satoshi KAMEI* \\ Department of Liberal Arts, Tokyo University of Technology, 1404-1 Katakura, Hachioji, Tokyo 192-0982, Japan
}

Received March 6, 2012; final version accepted August 1, 2012

\begin{abstract}
In this paper, we consider constructibility of simplicial 3-balls. Examining 1-dimensional subcomplexes of a simplicial 3-ball is efficient to solve the decision problem whether the simplicial 3-ball is constructible or not. From the point of view, we consider the case where a simplicial 3-ball has spanning edges and present a sufficient condition for nonconstructibility.
\end{abstract}

KEYWORDS: simplicial complex, shellability, constructibility

\section{Introduction}

Shellability and constructibility are combinatorial concepts relating to decompositions of simplicial balls. Since each shellable simplicial ball is constructible, the concepts form a hierarchy. Therefore examining constructibility of a simplicial ball is useful to decide whether the simplicial ball is shellable or not.

In [4], Hachimori presented a necessary and sufficient condition for constructibility of a reduced 3-ball which has no interior vertices. Further he proved that the condition is still necessary and sufficient in the case where a reduced 3-ball has at most two interior vertices in [5]. In [6], the author studied the case where a 3-ball has interior vertices and no edge which connects interior vertices, and presented a sufficient condition for nonconstructibility. In this paper, we deal with a more general case.

First, we recall the definition of constructibility of pure simplicial complexes. See [3] for the terminology of simplicial complexes. Further see [7] and [8] for the definition of shellability.

Definition. A pure $d$-dimensional simplicial complex $C$ is constructible if

(1) $C$ is a simplex, or

(2) there exist $d$-dimensional constructible subcomplexes $C_{1}$ and $C_{2}$ such that $C=C_{1} \cup C_{2}$ and that $C_{1} \cap C_{2}$ is a $(d-1)$-dimensional constructible simplicial complex.

We consider a decomposition of a pure $d$-dimensional constructible simplicial complex $C$ as follows. First, set $C^{(1)}=C$. We split $C^{(i)}$ into two $d$-dimensional constructible simplicial complexes $C_{1}^{(i)}$ and $C_{2}^{(i)}$ such that $C_{1}^{(i)} \cap C_{2}^{(i)}$ is a $(d-1)$-dimensional constructible simplicial complex. If there remains a $d$-dimensional constructible simplicial complex which is not a simplex, we denote it by $C^{(i+1)}$ and proceed to the $(i+1)$-th step. Since $C$ is constructible, there exists such a sequence of splittings and $C$ is finally split into simplices in finite steps. We reverse the sequence of splittings, then $C$ is constructed of simplices step by step. A sequence of gluings obtained in this way is called a construction of $C$.

A simplicial 3-ball $B$ is reduced if each 2-face which is not contained in $\partial B$ has at most one edge of $\partial B$. In [4], Hachimori introduced two reductional operations to obtain a reduced 3-ball $B_{2}$ from any simplicial 3-ball $B_{1}$. Further, he claimed that $B_{1}$ is constructible if and only if $B_{2}$ is constructible. As pointed out in [1], Hachimori's proof for the "if" part of the claim contains a gap. However, there is no gap in his proof for the "only if" part of that. Thus, if $B_{2}$ is nonconstructible, then $B_{1}$ is also nonconstructible. Therefore, it is essential to consider a sufficient condition for nonconstructibility of reduced 3-balls.

A vertex of $B$ not contained in $\partial B$ is called an interior vertex of $B$. An edge is called an interior edge of $B$ if both end vertices of the edge are interior vertices of $B$. Notice that an edge which has an end vertex contained in $\partial B$ is not called an interior edge, even though the edge is not contained in $\partial B$. An edge of $B$ is called spanning if the edge is not contained in $\partial B$ and both end vertices of the edge are contained in $\partial B$. A 1-dimensional simplicial complex which consists of interior vertices and interior edges of $B$ is called an interior graph of $B$. If an interior graph is maximal in $B$, the interior graph is called the maximal interior graph of $B$.

* Corresponding author. E-mail: kamei@cs.teu.ac.jp 
Let $C_{1}$ be a simplicial complex and $C_{2}$ be a subcomplex of $C_{1}$. In the followings, we denote by $\operatorname{Star}_{C_{1}} C_{2}$ the closed star neighbourhood of $C_{2}$ with respect to $C_{1}$.

Definition. A spanning edge $e$ is strict if there is no connected component $\Gamma$ of the maximal interior graph of $B$ such that both end vertices of $e$ are contained in exactly one connected component of $\partial B \cap \operatorname{Star}_{B} \Gamma$.

Notice that the definition agrees with the one in [6] if $B$ has no interior edge.

Now we state the main theorem.

Theorem 2.3. If all the spanning edges of a reduced 3-ball are strict, then the 3-ball is nonconstructible.

In Section 2, we prepare some technical lemmas and prove the main theorem. In Section 3, we see two examples.

\section{Main Argument}

First, we prepare technical lemmas needed in the proof of Theorem 2.3.

Lemma 2.1. Let $D$ be a simplicial 2-ball and $V$ be the set of the vertices of $D$. Let $W$ be a subset of the vertices of $\partial D$. We denote by $I$ the 1-dimensional maximal subcomplex of $D$ the vertices of which are the elements of $V \backslash W$. If $I$ is connected and every edge of $D$ connecting vertices of $W$ is contained in $\partial D$, then $\operatorname{Star}_{D} I$ coincides with $D$.

Proof. There is no 2-simplex of $D$ all vertices of which are contained in $W$, if otherwise there exists an edge which is not contained in $\partial D$ and connects vertices of $W$. Thus, for each vertex in $W$, there exists an edge which connects the vertex and a vertex in $V \backslash W$. Therefore all 2-simplices of $D$ are contained in $\operatorname{Star}_{D} I$ and the statement follows.

In the next lemma, we follow the symbols $D, V, W$ and $I$ in Lemma 2.1.

Lemma 2.2. Assume that I has more than one connected components. Then there exists an edge which is not contained in $\partial D$ and connects vertices of $W$.

Proof. Let $\Gamma_{1}$ and $\Gamma_{2}$ be connected components of $I$. Since $\left\{\operatorname{Star}_{D} \Gamma_{1}\right\} \cap \Gamma_{2}$ is empty, $\operatorname{Star}_{D} \Gamma_{1}$ dose not coincide with $D$. Further, all vertices of $\left\{\operatorname{Star}_{D} \Gamma_{1}\right\} \cap \overline{\left\{D \backslash \operatorname{Star}_{D} \Gamma_{1}\right\}}$ are contained in $W$. Thus there exists an edge which satisfies the conditions. See Fig. 1.

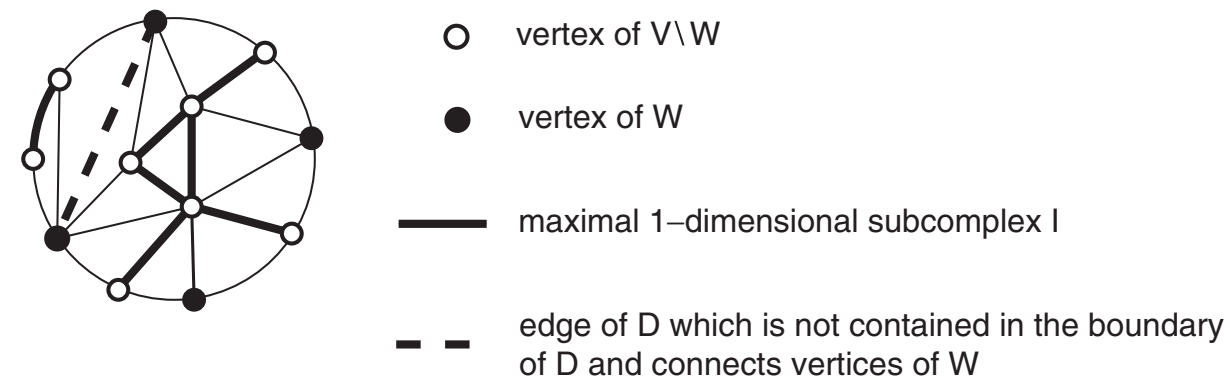

Fig. 1.

Further, we prepare some notions for the proof of Theorem 2.3. Let $D$ be a simplicial 2-ball which is a subcomplex of a simplicial 3-ball. Assume that $D$ contains spanning edges of the 3-ball. A spanning edge $e$ is outermost if $e$ cuts off a simplicial 2-ball $\Delta$ which contains no spanning edge except $e$ from $D$. The simplicial 2-ball $\Delta$ is called an outermost disk of $D$.

Let $B$ be a constructible 3-ball. We fix a construction of $B$. Let $B^{\prime}, B_{1}^{\prime}$ and $B_{2}^{\prime}$ be subcomplexes of $B$ such that $B^{\prime}=B_{1}^{\prime} \cup B_{2}^{\prime}$ is part of the construction of $B$. The 2-ball $B_{1}^{\prime} \cap B_{2}^{\prime}$ is called a divide. Notice that the interior of a divide contains no vertices of $\partial B$.

Now we prove the main theorem.

Theorem 2.3. If all the spanning edges of a reduced 3-ball are strict, then the 3-ball is nonconstructible.

Proof. Let $B$ be a reduced 3-ball which satisfies the hypothesis. We assume that $B$ is constructible and fix a construction of $B$. As in the definition of construction, we consider a decomposition of $B$ and denote subcomplexes of $B$ as follows. First, we set $B^{(1)}=B$. At each step of the decomposition, a 3-ball $B^{(i)}$ is split into two 3-balls $B_{1}^{(i)}$ and $B_{2}^{(i)}$ so that $B^{(i)}=B_{1}^{(i)} \cup B_{2}^{(i)}$ is part of the construction of $B$. Let $D^{(i)}=B_{1}^{(i)} \cap B_{2}^{(i)}$, so that $D^{(i)}$ is a divide.

We choose the step $i_{0}$ so that for the first time throughout the decomposition spanning edges appear on the boundaries of split 3-balls. Thus the spanning edges are contained in $D^{\left(i_{0}\right)}$. We choose an outermost spanning edge $e$ which cuts off an outermost disk $\Delta$ from $D^{\left(i_{0}\right)}$. Let $I$ be the maximal interior graph of $B$. Then $\Delta \cap I$ is connected, if 
otherwise there would be a spanning edge except $e$ in $\Delta$ from Lemma 2.2 and it contradicts the assumption that $\Delta$ is outermost. Let $\Gamma^{\left(i_{0}\right)}$ be $\Delta \cap I$ and $P^{\left(i_{0}\right)}$ be $\overline{\partial \Delta \backslash e}$. From Lemma 2.1, $\operatorname{Star}_{\Delta} \Gamma^{\left(i_{0}\right)}$ coincides with $\Delta$. Thus the path $P^{\left(i_{0}\right)}$ is contained in $\partial B^{\left(i_{0}\right)} \cap \operatorname{Star}_{B^{\left(i_{0}\right)}} \Gamma^{\left(i_{0}\right)}$.

If all vertices of $P^{\left(i_{0}\right)}$ are contained in $\partial B$, all edges of $P^{\left(i_{0}\right)}$ are also contained in $\partial B$ since $\partial B^{\left(i_{0}\right)}$ contains no spanning edges. In this case, the spanning edge $e$ is not strict and it contradicts the assumption that all spanning edges are strict. Thus there exist vertices of $P^{\left(i_{0}\right)}$ which are not contained in $\partial B$. Let $v_{1}$ and $v_{2}$ be the end vertices of $e$. In the following, we construct paths $P^{(i)}$ which connect $v_{1}$ and $v_{2}$ on $\partial B^{(i)}$ from $i=i_{0}-1$ to 1 inductively. Also we construct a connected interior graph $\Gamma^{(i)}$ in $B^{(i)}$ so that $\partial B^{(i)} \cap \operatorname{Star}_{B^{(i)}} \Gamma^{(i)}$ contains $P^{(i)}$, and deduce a contradiction.

We start with $P^{\left(i_{0}\right)}$ and $\Gamma^{\left(i_{0}\right)}$. If $P^{\left(i_{0}\right)} \cap\left\{D^{\left(i_{0}-1\right)} \backslash \partial D^{\left(i_{0}-1\right)}\right\}$ is empty, we set $P^{\left(i_{0}-1\right)}=P^{\left(i_{0}\right)}$ and $\Gamma^{\left(i_{0}-1\right)}=\Gamma^{\left(i_{0}\right)}$. Otherwise, we choose two vertices of $P^{\left(i_{0}\right)} \cap \partial D^{\left(i_{0}-1\right)}$, say $w_{1}$ and $w_{2}$, which are the nearest to $v_{1}$ and $v_{2}$ on $P^{\left(i_{0}\right)}$ respectively. If $P^{\left(i_{0}\right)} \cap \partial D^{\left(i_{0}-1\right)}$ contains $v_{1}$ and $v_{2}$, we set $w_{j}=v_{j}$ for $j=1,2$. Notice that there exist more than one vertices which are contained in $P^{\left(i_{0}\right)} \cap \partial D^{\left(i_{0}-1\right)}$ because $P^{\left(i_{0}\right)} \cap\left\{D^{\left(i_{0}-1\right)} \backslash \partial D^{\left(i_{0}-1\right)}\right\}$ is not empty and $\partial D^{\left(i_{0}-1\right)}$ does not separate $v_{1}$ and $v_{2}$ on $\partial B^{\left(i_{0}-1\right)}$. We switch the subpath of $P^{\left(i_{0}\right)}$ with a connected subcomplex of $\partial D^{\left(i_{0}-1\right)}$ both of which connect $w_{1}$ and $w_{2}$. Although there are two connected subcomplexes of $\partial D^{\left(i_{0}-1\right)}$ the end vertices of which are $w_{1}$ and $w_{2}$, we may choose either of them. We denote by $P^{\left(i_{0}-1\right)}$ the constructed path, so that $P^{\left(i_{0}-1\right)}$ connects $v_{1}$ and $v_{2}$ on $\partial B^{\left(i_{0}-1\right)}$. See Fig. 2. Next, we construct $\Gamma^{\left(i_{0}-1\right)}$. Since there are no spanning edges which are contained in $D^{\left(i_{0}-1\right)}$, $D^{\left(i_{0}-1\right)} \cap I$ is connected. Then $\operatorname{Star}_{D^{\left(i_{0}-1\right)}}\left\{D^{\left(i_{0}-1\right)} \cap I\right\}$ coincides with $D^{\left(i_{0}-1\right)}$ from Lemma 2.1. Further, $\left\{D^{\left(i_{0}-1\right)} \cap I\right\} \cup$ $\Gamma^{\left(i_{0}\right)}$ is connected because there exists a vertex of $P^{\left(i_{0}\right)}$ which is not contained in $\partial B$. We set $\Gamma^{\left(i_{0}-1\right)}=$ $\left\{D^{\left(i_{0}-1\right)} \cap I\right\} \cup \Gamma^{\left(i_{0}\right)}$. Obviously, the path $P^{\left(i_{0}-1\right)}$ is contained in $\partial B^{\left(i_{0}-1\right)} \cap \operatorname{Star}_{B^{\left(i_{0}-1\right)}} \Gamma^{\left(i_{0}-1\right)}$.

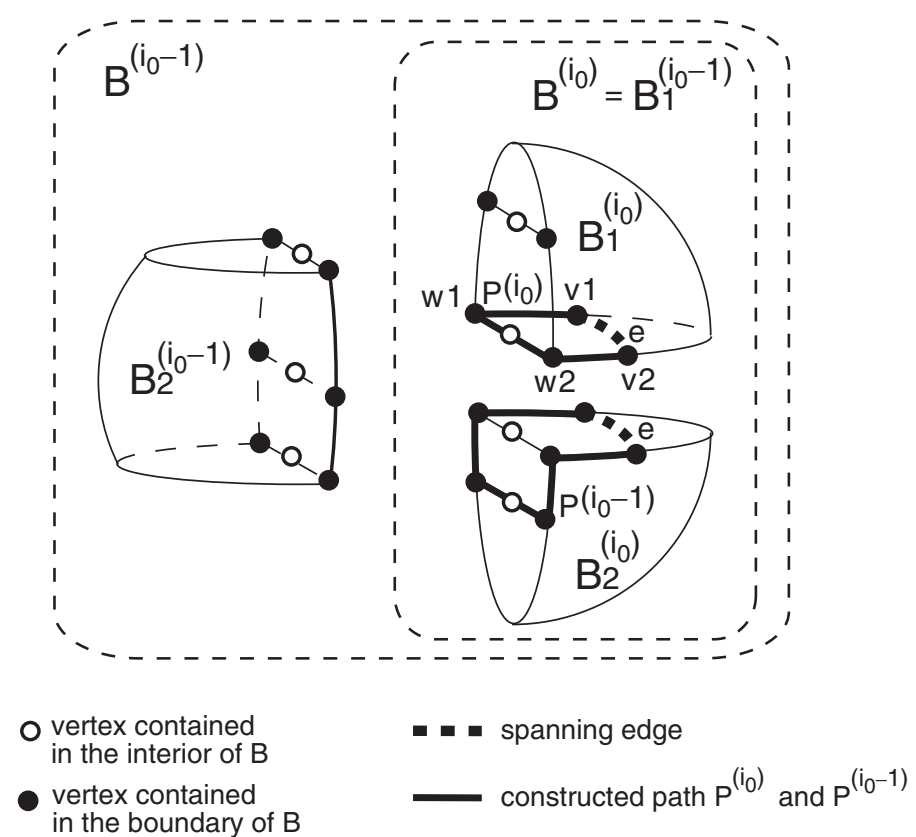

Fig. 2.

Continuously, we construct $P^{(j)}$ and $\Gamma^{(j)}$ from $j=i_{0}-1$ to 1 . Then $P^{(1)}$ is a path which connects $v_{1}$ and $v_{2}$ on $\partial B$. Also $P^{(1)}$ is contained in $\partial B \cap \operatorname{Star}_{B} \Gamma^{(1)}$. Thus $e$ is not strict and it contradicts our assumption.

\section{Examples}

In this section, we see two examples. The first example is a 3-ball which satisfies the hypothesis of Theorem 2.3.

Example 3.1. We construct an example based on Example 4.1 in [6]. First, we recall the example.

Consider the 3-ball which is depicted in the left side of Fig. 3. The walls of the ball are made of one layer of cubes. There are two tunnels one of which connects the upper space and the lower floor, and the other of which connects the lower space and the upper floor. The 3-ball is known as "Bing's house with two rooms" described in [2].

We triangulate the 3-ball as follows. First, triangulate the 2-skeleton of the 3-ball. Next, add an interior vertex in each cube, and construct a cone from the vertex over the triangulated boundary of each cube. Notice that there is an appropriate triangulation of the 2-skeleton of the 3-ball such that the triangulated 3-ball is reduced. See [4] and [6] for more details. The constructed simplicial 3-ball satisfies the hypothesis of Theorem 3.1 in [6]. Thus the simplicial 3-ball is not constructible. 


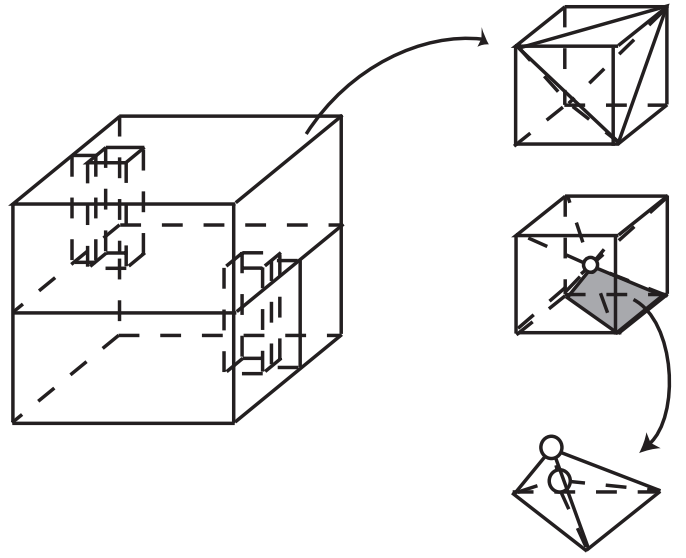

(1) Triangulate the boundary of each cube.

(2) Add an interior vertex in the interior of each cube and construct a cone from the vertex.

(3) Add an interior vertex in the interior of each tetrahedron and construc a cone from the vertex.

Fig. 3.

Now we add an interior vertex in each tetrahedron and construct a cone from the vertex over the boundary of each tetrahedron. In each cube, there exists exactly one connected component of the maximal interior graph of the 3-ball. The constructed simplicial 3-ball satisfies the hypothesis of Theorem 2.3, thus the 3-ball is still nonconstructible.

The second example is a shellable 3-ball which has a strict spanning edge and nonstrict spanning edges.

Example 3.2. This example is constructed as indicated in Fig. 4. First, we consider a solid trigonal pillar "3-4-56-7-8." Add edges "3-8," "5-7" and "4-6," then the boundary of the pillar is triangulated. Add vertices "1" and "2" in the interiors of 2-simplices " $3-4-5$ " and "6-7-8" respectively. Connect the vertex " 1 " and the vertices " 4,5 , 6," and the vertex "2" and "7, 8, 9" by edges respectively. Add a spanning edge "1-2" and triangulate the pillar as Fig. 4(a). Put a simplicial 1-sphere "9-10-11" such that the pillar goes through the 1-sphere. Connect the point " 9 " and the points " $3,5,6,8$ " by edges respectively. Also connect the point " 10 " and the points " $3,4,6,7$," the point " 11 " and the points " $4,5,7,8$ " in the same way. Fill tetrahedra which have the edges added at the previous step and the edges on the

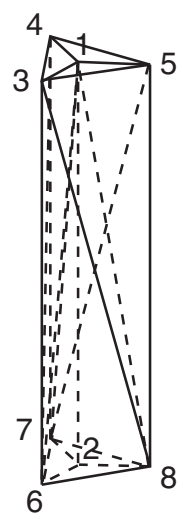

(a)

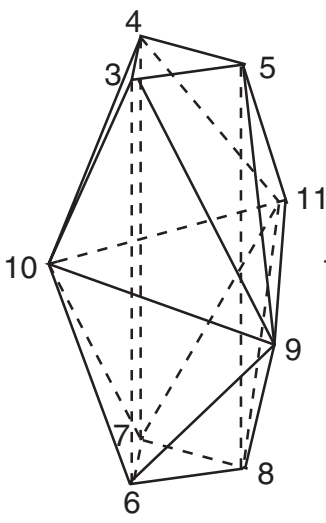

(b)

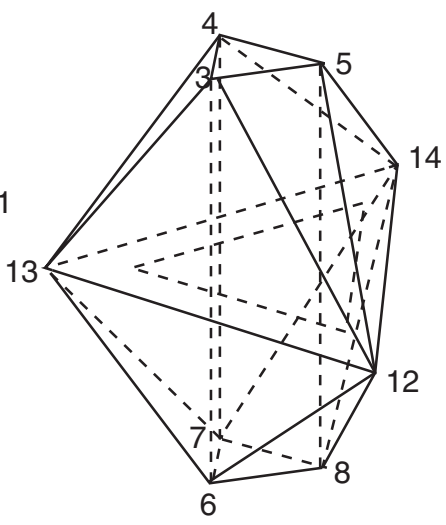

(c)

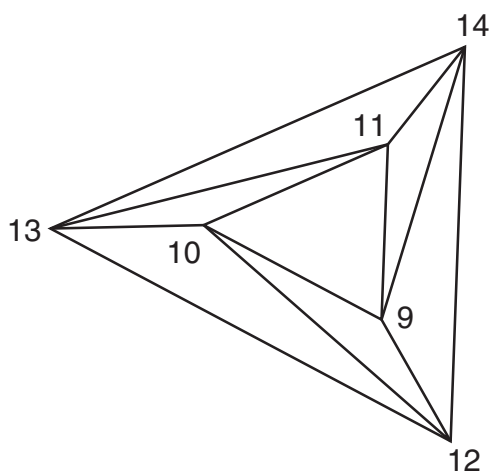

(d)

Fig. 4. 
boundary of the pillar. Further put a simplicial 1-sphere "12-13-14" as Fig. 4(d). Connect the point "12" and the points " $3,5,6,8,9,10$," the point " 13 " and the points " $3,4,6,7,10,11$," and the point " 14 " and the points " $4,5,7,8,9,11$ " by edges respectively as Figs. 4(c) and 4(d). Fill tetrahedra the same as the previous step.

The constructed 3-ball is reduced and has exactly one connected maximal interior graph "9-10-11." Further the 3ball has one strict spanning edge "1-2" and three nonstrict spanning edges "3-6," "4-7," "5-8." We can easily check that the 3-ball is shellable thus it is also constructible. This example suggests that we cannot relax the latter hypothesis of Theorem 2.3, that is, all spanning edges of a reduced 3-ball are strict.

The following is the list of all facets of this example.

$\begin{array}{lllll}\{1,2,6,7\} & \{1,2,7,8\} & \{1,2,6,8\} & \{1,3,5,8\} & \{1,3,6,8\} \\ \{1,3,4,6\} & \{1,4,6,7\} & \{1,4,5,7\} & \{1,5,7,8\} & \\ \{5,8,9,11\} & \{3,5,8,9\} & \{3,6,8,9\} & \{3,6,9,10\} & \{3,4,6,10\} \\ \{4,6,7,10\} & \{4,7,10,11\} & \{4,5,7,11\} & \{5,7,8,11\} & \\ \{3,4,10,13\} & \{3,10,12,13\} & \{3,9,10,12\} & \{3,5,9,12\} & \{5,9,12,14\} \\ \{5,9,11,14\} & \{4,5,11,14\} & \{4,11,13,14\} & \{4,10,11,13\} & \\ \{6,7,10,13\} & \{6,10,12,13\} & \{6,9,10,12\} & \{6,8,9,12\} & \{8,9,12,14\} \\ \{8,9,11,14\} & \{7,8,11,14\} & \{7,11,13,14\} & \{7,10,11,13\} & \end{array}$

\section{Acknowledgement}

The author would like to thank Professor Masahiro Hachimori for his helpful advice and discussions for the construction of Example 3.2.

\section{REFERENCES}

[1] Benedetti, B., and Ziegler, G. M., "On locally constructible spheres and balls," Acta Mathematica, 206: $205-243$ (2011).

[2] Bing, R. H., Some aspects of the topology of 3-manifolds related to the Poincare conjecture, in Lectures on Modern Mathematics II (ed. T. L. Saaty), 93-128, Wiley, New York (1964).

[3] Björner, A., Topological methods, in Handbook of Combinatorics (eds. Graham, R., Grötschel, M., and Lovász, L.), 1819-1872, North-Holland/Elsevier, Amsterdam (1995).

[4] Hachimori, M., "Nonconstructible simplicial balls and a way of testing constructibility," Discrete \& Computational Geometry, 22: 223-230 (1999).

[5] Hachimori, M., "Deciding constructibility of 3-balls with at most two interior vertices," Discrete Mathematics, 232: 47-58 (2001).

[6] Kamei, S., "Deciding nonconstructibility of 3-balls with spanning edges and interior vertices," Discrete Mathematics, 307: 3201-3206 (2007).

[7] Ziegler, G. M., Lectures on Polytopes, Springer-Verlag, New York (1995).

[8] Ziegler, G. M., “Shelling polyhedral 3-balls and 4-polytopes,” Discrete \& Computational Geometry, 19: 159-174 (1998). 